\title{
A simple and complete model theory for intensional and extensional untyped $\lambda$-equality
}

\author{
Michael Gabbay * \\ Department of Philosophy, Kings College London \\ http://www.gabbay.org.uk
}

\begin{abstract}
We present a sound and complete model theory for theories of $\beta$-reduction with or without $\eta$-expansion. We then show in what conditions we obtain models of $\beta$-equality and $\beta \eta$-equality.
\end{abstract}

\section{Introduction}

We extend the method of [GG10] by which we are able to interpret $\lambda$-terms compositionally on 'possible world' structures. The simplicity of the structures is striking, moreover, they provide us with a surprising richness of interpretations of function abstraction and application. Our primary goal is to show how the models can differentiate between extensional and intensional $\lambda$-equality, and provide semantic characterisation (i.e. completeness) theorems for both. We shall then hint at how richer $\lambda$-languages can be interpreted.

The key idea in this paper is a class of models, presented in Section 2.2 although an important syntactic consideration is required first in Section 2.1. These ideas bear some similarity to the reduction models of Sel03 in that they get us as far as $\lambda$-reduction only. Then, in Section 4 we make use of the results of the earlier sections to provide a characterisation theorem for $\lambda$-equality (both with and without $\eta$-equality, i.e. extensional and intensional). We can then use this characterisation to comment on the issue of order-incompleteness.

\section{The models, computation, logic}

\subsection{The language and logic}

Definition 2.1. Fix a countably infinite set of variables.

Define a language $\mathcal{L}_{\lambda}$ of $\lambda$-terms by:

$$
\mathrm{t}::=\mathrm{x}|\lambda \mathrm{x} \cdot \mathrm{t}| \mathrm{t} \cdot \mathrm{t}
$$

$\lambda \mathrm{x}$ binds in $\lambda \mathrm{x}$.t. For example, $\mathrm{x}$ is bound (not free) in $\lambda \mathrm{x} . \mathrm{x} \cdot \mathrm{y}$.

We write $\mathrm{t}[\mathrm{x} / \mathrm{s}]$ for the usual capture-avoiding substitution. For example, $(\lambda z \cdot y)[y / x]=\lambda z \cdot x$, and $(\lambda x \cdot y)[y / x]=\lambda z \cdot x$ where $z$ is an arbitrary fresh variable.

We write $\mathrm{t}[\mathrm{x}:-\mathrm{s}]$ for the (unusual) non-capture avoiding substitution. For example, $(\lambda \mathrm{x} \cdot \mathrm{x})[\mathrm{x}:-\mathrm{y}]=\lambda \mathrm{x} \cdot \mathrm{y},(\lambda \mathrm{x} \cdot \mathrm{y})[\mathrm{y}:-\mathrm{x}]=\lambda \mathrm{x} \cdot \mathrm{x}$ and $\mathrm{x}[\mathrm{y}:-\mathrm{t}]=\mathrm{x}$.

* Supported by IFCoLog Hilbert-Bernays Project 
Let $\mathrm{x}$ occur free only once in $\mathrm{t}$.

( $\beta) \quad \mathrm{t}[\mathrm{x}:-(\lambda \mathrm{x} . \mathrm{s}) \cdot \mathrm{r}] \longrightarrow \longrightarrow_{\Gamma} \mathrm{t}[\mathrm{x}:-\mathrm{s}[\mathrm{x} / \mathrm{r}]] \quad($ ass $) \quad \mathrm{t}[\mathrm{x}:-\mathrm{s}] \longrightarrow_{\Gamma} \mathrm{t}[\mathrm{x}:-\mathrm{r}] \quad(\langle\mathrm{s}, \mathrm{r}\rangle \in \Gamma)$

$(\alpha) \mathrm{t}[\mathrm{x}:-\lambda \mathrm{y} \cdot \mathrm{s}] \longrightarrow_{\Gamma} \mathrm{t}[\mathrm{x}:-\lambda \mathrm{z} . \mathrm{s}[\mathrm{y} / \mathrm{z}]]$

Figure 1: $\lambda$-reduction for $\mathcal{L}_{\lambda}$

We now turn to $\lambda$-reduction. We shall define a basic relation on terms that follows the familiar reduction rule of $\beta$-contraction. To help with the completeness theorem of Section 3 we will need to consider a conservative extension of the familiar $\lambda$-calculus (Definition 2.4). To facilitate the proof that this extension really is conservative (Theorem 2.7), we present the $\lambda$-calculus in the non-axiomatic style of [HS08, Def. 1.24]. It is important for us to consider not merely the relation of $\lambda$-reduction, but a relation of $\lambda$-reduction with assumptions. We do this so we can consider the theory of $\lambda$-equality as an extension of the theory of $\lambda$-reduction. Our models shall initially model only be sound for $\beta$-reduction and $\eta$-expansion. We can then begin our characterisation of $\beta \eta$-equality by considering the subclass of these models that also validate $\beta$-expansion and $\eta$-reduction. We therefore need to define some basic, and familiar, rules of $\lambda$-reduction but allow for a set of assumed additional reductions (we do not seek to study the syntactic properties of a general calculus of $\lambda$-reduction with assumptions in this paper).

Definition 2.2. Let $\Gamma$ be a set of pairs of terms of $\mathcal{L}_{\lambda}$. We define a reduction relation $\longrightarrow_{\Gamma}$ on terms of $\mathcal{L}_{\lambda}$ using Figure 1. A derivation is a sequence of terms $t_{1}, \ldots, t_{n}$ such that $\mathrm{t}_{i} \longrightarrow_{\Gamma} \mathrm{t}_{i+1}$ for each $1 \leq i<n$.

Remark 2.3. If $\Gamma=\varnothing$ then $\longrightarrow_{\Gamma}$ is the familiar relation of untyped $\beta$-reduction.

Definition 2.4. Define $\mathcal{L}_{\lambda}^{*}$ by: $\quad$ t $::=\mathrm{x} \mid \lambda$ x.t $|\mathrm{t} \cdot \mathrm{t}| \mathrm{t} * \mathrm{t}$

Definition 2.5. Let $\Gamma$ be a set of pairs of terms of $\mathcal{L}_{\lambda}\left(\right.$ not $\left.\mathcal{L}_{\lambda}^{*}\right)$. Define a reduction relation $\Longrightarrow_{\Gamma}$ on terms of $\mathcal{L}_{\lambda}^{*}$ using Figure 2. Again, a derivation is a sequence of terms $t_{1}, \ldots, t_{n}$ such that $\mathrm{t}_{i} \Longrightarrow_{\Gamma} \mathrm{t}_{i+1}$ for each $1 \leq i<n$.

Remark 2.6. Notice that we do not allow terms unique to $\mathcal{L}_{\lambda}^{*}$ to be assumptions in derivations. There are two reasons for this. Firstly, the paper is initially concerned with characterising reduction and equality only in the more familiar language $\mathcal{L}_{\lambda}$, and $\mathcal{L}_{\lambda}^{*}$ is merely a means to that end. Allowing assumed reductions for $\mathcal{L}_{\lambda}^{*}$ adds needless complexity to Theorem 2.7. Secondly, a further aim is to show that $\beta$-equality (i.e. $\beta$-expansion and contraction) is complete for models with a nontrivial ordering relation on them. The fact that $\beta$-equality holds for $\mathcal{L}_{\lambda}$ but not for $\mathcal{L}_{\lambda}^{*}$ helps to emphasise this.

Theorem 2.7. If $t_{1}$ and $t_{2}$ are terms of $\mathcal{L}_{\lambda}$ then $t_{1} \Longrightarrow_{\Gamma} t_{2}$ implies $t_{1} \longrightarrow_{\Gamma} t_{2}$. In other words $\Longrightarrow_{\Gamma}$ is conservative over $\longrightarrow_{\Gamma}$.

Proof. See the technical appendix A.1

\section{$2.2 \quad$ Frames and interpreting $\lambda$-terms}

Definition 2.8. If $W$ is a set, write $\mathcal{P}(W)$ for the set of subsets of $W$.

An intensional frame $F$ is a 4 -tuple $(W, \bullet, \circ, H)$ where: 
Let $\mathrm{x}$ occur free only once in $\mathrm{t}$.

$$
\begin{aligned}
& \text { ( } \beta) \quad \mathrm{t}[\mathrm{x}:-(\lambda \mathrm{x} . \mathrm{s}) \cdot \mathrm{r}] \Longrightarrow_{\Gamma} \mathrm{t}[\mathrm{x}:-\mathrm{s}[\mathrm{x} / \mathrm{r}]] \quad \text { (ass) } \mathrm{t}[\mathrm{x}:-\mathrm{s}] \Longrightarrow_{\Gamma} \mathrm{t}[\mathrm{x}:-\mathrm{r}]\langle\mathrm{s}, \mathrm{r}\rangle \in \Gamma \\
& \text { ( } \alpha) \mathrm{t}[\mathrm{x}:-\lambda \mathrm{y} . \mathrm{s}] \Longrightarrow_{\Gamma} \mathrm{t}[\mathrm{x}:-\lambda \mathrm{z} . \mathrm{s}[\mathrm{y} / \mathrm{z}]] \\
& \left(\beta^{*}\right) \quad \mathrm{t}[\mathrm{x}:-(\lambda \mathrm{x} . \mathrm{s}) * \mathrm{r}] \Longrightarrow_{\Gamma} \mathrm{t}[\mathrm{x}:-\mathrm{s}[\mathrm{x} / \mathrm{r}]] \quad \text { (sub) } \quad \mathrm{t}[\mathrm{x}:-\mathrm{s} \cdot \mathrm{r}] \Longrightarrow_{\Gamma} \mathrm{t}[\mathrm{x}:-\mathrm{s} * \mathrm{r}] \\
& \left(\eta^{*}\right) \mathrm{t}[\mathrm{x}:-\mathrm{s}] \Longrightarrow_{\Gamma} \mathrm{t}[\mathrm{x}:-\lambda \mathrm{y} \cdot(\mathrm{s} * \mathrm{y})] \quad(\mathrm{y} \text { not free in } \mathrm{s})
\end{aligned}
$$

Figure 2: $\lambda$-reduction for $\mathcal{L}_{\lambda}^{*}$

$-W$ a set of worlds,

- $\bullet$ and $\circ$ are functions from $W \times W$ to $\mathcal{P}(W)$ such that $\bullet \subseteq \circ$.

$-H \subseteq \mathcal{P}(W)$.

Remark 2.9. Subsets of $W$ will serve as denotations of $\lambda$-terms (Definition 2.12 and $H \subseteq$ $\mathcal{P}(W)$ (' $H$ ' for 'Henkin') plays a similar role to the structure of Henkin models for higher-order logic BBK04, Hen50, Sha00. This makes our completeness results possible and is a famous issue for second- and higher-order logics. Powersets are too large; for completeness results to be possible we must cut them down — at least when we quantify. This is why in Definition 2.12 . the binders restrict quantification from $\mathcal{P}(W)$ down to $H$.

The reader familiar with modal logic can think of $\bullet$ and $\circ$ as ternary 'accessibility relations' $R_{\bullet}$ and $R_{\circ}$ such that $R_{\bullet} w_{1} w_{2} w_{3}$ if and only if $w_{3} \in w_{1} \bullet w_{2}$ (and similarly for $R_{\circ}$ ). We can also think of $\bullet$ and $\circ$ as non-deterministic 'application' operations, but note that intensional frames are not applicative structures - an applicative structure would map $W \times W$ to $W$, whereas in the case of intensional frames, $W \times W$ maps to $\mathcal{P}(W)$.

Definition 2.10. Let $F=(W, \bullet, \circ, H)$ be an intensional frame and $S_{1}, S_{2} \subseteq W$ and $w \in W$. Then the functions $\bullet$ and $\circ$ induce functions from $W \times \mathcal{P}(W)$ and $\mathcal{P}(W) \times \mathcal{P}(W)$ to $\mathcal{P}(W)$ by: $w \bullet S=\bigcup\left\{w \bullet w^{\prime} \mid w^{\prime} \in S\right\}$ and $S_{1} \bullet S_{2}=\bigcup\left\{w_{1} \bullet w_{2} \mid w_{1} \in S_{1}, w_{2} \in S_{2}\right\}$ (and similarly for $\circ$ ).

Definition 2.11. Suppose $F=(W, \bullet, \circ, H)$ is a frame. A valuation (to $F$ ) is a map from variables to sets of worlds (elements of $\mathcal{P}(W)$ ) that are in $H . v$ will range over valuations.

If $\mathrm{x}$ is a variable, $h \in H$, and $v$ is a valuation, then write $v[\mathrm{x} \mapsto h]$ for the valuation mapping $\mathrm{x}$ to $h$ and mapping $\mathrm{y}$ to $v(\mathrm{y})$ for any other $\mathrm{y}$.

Definition 2.12. Define the denotation of $t$ inductively by:

$$
\begin{aligned}
& \llbracket \mathrm{x} \rrbracket^{v}=v(\mathrm{x}) \quad \llbracket \mathrm{t} \cdot \mathrm{s} \rrbracket^{v}=\llbracket \mathrm{t} \rrbracket^{v} \bullet \llbracket \mathrm{s} \rrbracket^{v} \quad \llbracket \mathrm{t} * \mathrm{~s} \rrbracket^{v}=\llbracket \mathrm{t} \rrbracket^{v} \circ \llbracket \mathrm{s} \rrbracket^{v} \\
& \llbracket \lambda \mathrm{x} . \mathrm{t} \rrbracket^{v}=\left\{w \mid w \circ h \subseteq \llbracket \mathrm{t} \rrbracket^{v[\mathrm{x} \mapsto h]} \text { for all } h \in H\right\}
\end{aligned}
$$

Remark 2.13. By elementary set theory: $\llbracket \lambda \mathrm{x} . \mathrm{t} \rrbracket^{v}=\bigcap_{h \in H}\left\{w \mid w \circ h \subseteq \llbracket \mathrm{t} \rrbracket^{v[\mathrm{x} \mapsto h]}\right\}$

We are particularly interested in frames where the denotation of every $\lambda$-term is a member of $H$. This is because Definition 2.12 interprets $\lambda$ as a kind of quantifier over all members of $H$. $\beta$-reduction then becomes a form of universal instantiation and so requires that every possible instantiation (i.e. every term denotation) is a member of $H$.

Definition 2.14. A frame is faithful when for every $v$ and every $\mathrm{t}, \llbracket \mathrm{t} \rrbracket^{v} \in H$.

Lemma 2.15. 1. If $\mathrm{x}$ is not free in $\mathrm{t}$, then for any $h \in H$, $\llbracket \mathrm{t} \rrbracket^{v}=\llbracket \mathrm{t} \rrbracket^{v[\mathrm{x} \mapsto h]}$. 


\section{2. $\llbracket \mathrm{t}[\mathrm{x} / \mathrm{s}] \rrbracket^{v}=\llbracket \mathrm{t} \rrbracket^{v\left[\mathrm{x} \mapsto \llbracket \mathbb{s} \rrbracket^{v}\right]}$}

Proof. Both parts follow by easy inductions on $t$.

Theorem 2.16. If we interpret $\longrightarrow_{\Gamma}$ as subset inclusion then all the rules of Figure 2 are sound for faithful intensional frames.

Proof. By routine calculations from the definitions. See the technical appendix A.2

\subsection{Soundness}

Definition 2.17. - A model $M$ is a pair $\langle F, v\rangle$ where $F$ is a faithful intensional frame and $v$ is a valuation on $F$.

- Say a model is $\Gamma$-sensitive if $\llbracket \mathrm{t} \rrbracket^{v} \subseteq \llbracket \mathrm{s} \rrbracket^{v}$ for every $\langle\mathrm{t}, \mathrm{s}\rangle \in \Gamma$.

- Say a frame $F$ is $\Gamma$-sensitive if $\langle F, v\rangle$ is $\Gamma$-sensitive for every $v$.

Remark 2.18. We could have defined a model as a pair $\langle F, v\rangle$ where $F=\langle W, \bullet, \circ, H\rangle$ is a (possible not faithful) frame and $v$ is a valuation on $F$ such that $\llbracket \mathrm{t} \rrbracket^{v} \in H$ for every $\mathrm{t}$. But since the completeness theorem 3.9 holds for the stronger notion of a model we shall use that.

Lemma 2.19. • and $\circ$ are monotone. That is, $h_{1} \subseteq h_{2}$ implies $h \bullet h_{1} \subseteq h \bullet h_{2}$ and $h_{1} \bullet h \subseteq h_{2} \bullet h$ for any $h$, and similarly for $\circ$.

Lemma 2.20. If $\llbracket \mathrm{s} \rrbracket^{v} \subseteq \llbracket \mathrm{r} \rrbracket^{v}$ for all $v$ on some faithful $F$, then $\llbracket \mathrm{t}[\mathrm{x}:-\mathrm{s}] \rrbracket^{v} \subseteq \llbracket \mathrm{x}:-\mathrm{r} \rrbracket^{v}$

Theorem 2.21. $\mathrm{t} \Longrightarrow_{\Gamma} \mathrm{s}$ implies $\llbracket \mathrm{t} \rrbracket^{v} \subseteq \llbracket \mathrm{s} \rrbracket^{v}$ in all $\Gamma$-sensitive models $M$.

Proof. Theorem 2.16 entails that each rule of Figure 2 holds in all models, and by definition, if $\langle\mathrm{t}, \mathrm{s}\rangle \in \Gamma$ then $\llbracket \mathrm{t} \rrbracket^{v} \subseteq \llbracket \mathbf{s} \rrbracket^{v}$ in all $\Gamma$-sensitive models. The result then follows by Lemma 2.20 .

\section{Completeness for $\lambda$-reduction}

We must show that if $\mathrm{t}-\lrcorner_{\Gamma} \mathbf{s}$ then there is a $\Gamma$-sensitive model $M$ where $\llbracket \mathrm{t} \rrbracket^{v} \nsubseteq \llbracket \mathrm{s} \rrbracket^{v}$. We first show that $\mathrm{t} \Longrightarrow_{\Gamma} \mathrm{s}$ implies such an $M$ exists if $\mathrm{t}, \mathrm{s} \in \mathcal{L}_{\lambda}$, and then we appeal to Theorem 2.7.

First we add infinitely many new constant symbols $c_{1}, c_{2} \ldots$ to the language $\mathcal{L}_{\lambda}^{*}$. Since the language is countable we can enumerate its terms $t_{1}, t_{2} \ldots$, which may contain the new constants, and the new constants alone $\mathrm{c}_{1}, \mathrm{c}_{2} \ldots$ We describe a one-one function $f$ from terms to constants.

$f\left(\mathrm{t}_{i}\right)=\mathrm{c}_{j}$ where $j$ is the least number such that $j>i$ and $\mathrm{c}_{j}$ does not occur in $\mathrm{t}_{i}$ nor is the value under $f$ of any $\mathrm{t}_{k}$ for $k<i$.

Thus $f$ is a one-one function that assigns a distinct 'fresh' constant to each term of the language. Thus $f(\mathrm{t})$ is a constant that 'names' t. These play the role of witness constants in the construction of the canonical frame in Theorem 3.7. The $f(\mathrm{t})$ also help us carry out inductions on the size of $\lambda$-terms, as $\mathrm{t}[\mathrm{x} / f(\mathrm{~s})]$ is smaller than $\lambda \mathrm{x}$.t even if $\mathrm{t}[\mathrm{x} / \mathrm{s}]$ might not be.

Definition 3.1. Let $\zeta_{f}=\{\langle\mathrm{t}, f(\mathrm{t})\rangle \mid \mathrm{t}$ is a term $\} \cup\{\langle f(\mathrm{t}), \mathrm{t}\rangle \mid \mathrm{t}$ is a term $\}$. Write $\mathrm{t} \Longrightarrow_{\Gamma}^{\zeta_{f}} \mathrm{~s}$ when $\zeta_{f} \subseteq \Gamma$ and $\mathrm{t} \Longrightarrow_{\Gamma} \mathbf{s}$.

Lemma 3.2. If $\mathrm{t} \Longrightarrow{ }_{\Gamma}^{\zeta_{f}} \mathrm{~s}$ and neither $\mathrm{s}$ or $\mathrm{t}$ contain any of the new constants $\mathrm{c}_{1}, \mathrm{c}_{2} \ldots$, then $\mathrm{t} \Longrightarrow_{\Gamma} \mathrm{s}$. 
Proof. $f$ is defined in terms of an enumeration such that $r$ always precedes $f(r)$. Thus if we repeatedly substitute each instance of $f(r)$ with $\mathrm{r}$ in a derivation, eventually all will be eliminated. But then instances of (ass) depending on $\zeta_{f}$ become trivial reductions $\mathrm{r} \Longrightarrow{ }_{\Gamma}^{\zeta_{f}} \mathrm{r}$ which can be removed without affecting the rest of the derivation. Certainly the first and final terms $\mathrm{t}$ and $\mathrm{s}$ are unaffected as they never contained any $f(\mathrm{r})$ in the first place.

Definition 3.3. If $\mathrm{t}$ is a term let $w_{\mathrm{t}}=\left\{\mathbf{s} \mid \mathrm{t} \Longrightarrow{ }_{\Gamma}^{\zeta_{f}} \mathbf{s}\right\}$. Thus $w_{\mathrm{t}}$ is the closure of $\mathrm{t}$ under $\Longrightarrow \stackrel{\zeta_{f}}{\Gamma}$.

Definition 3.4. Define the canonical $\lambda$-frame $F_{\lambda}=\left\langle W_{\lambda}, \bullet_{\lambda}, o_{\lambda}, H_{\lambda}\right\rangle$ :

$$
\begin{gathered}
W_{\lambda}=\left\{w_{\mathrm{t}} \mid \mathrm{t} \in \mathcal{L}_{\lambda}^{*}\right\} \quad H_{\lambda}=\left\{\left\{w_{\mathrm{s}} \in W_{\lambda} \mid \mathrm{t} \in w_{s}\right\} \mid \mathrm{t}, \mathbf{s} \in \mathcal{L}_{\lambda}^{*}\right\} \\
w_{\mathrm{t}} \bullet_{\lambda} w_{\mathrm{s}}=\left\{w \in W_{\lambda} \mid \mathrm{t} \cdot \mathrm{s} \in w\right\} \quad w_{\mathrm{t}} \circ_{\lambda} w_{\mathrm{s}}=\left\{w \in W_{\lambda} \mid \mathrm{t} * \mathbf{s} \in w\right\}
\end{gathered}
$$

Definition 3.5. Given $F_{\lambda}=\left\langle W_{\lambda}, \bullet_{\lambda}, \circ_{\lambda}, H_{\lambda}\right\rangle$ and a term $\mathrm{t}$ of $\mathcal{L}_{\lambda}^{*}$. Let $\|\mathrm{t}\|=\left\{w \in W_{\lambda} \mid \mathrm{t} \in w\right\}$. Note that $H_{\lambda}=\left\{\|\mathrm{t}\| \mid \mathrm{t} \in \mathcal{L}_{\lambda}^{*}\right\}$

Remark 3.6. Given $(s u b)$ it is easy to see that $\bullet_{\lambda} \subseteq \circ_{\lambda}$. To see how $\bullet_{\lambda}$ and $\circ_{\lambda}$ come apart consider $w_{\lambda \mathbf{y} \cdot(\mathbf{x} \cdot \mathbf{y})}, w_{\mathbf{z}}$ and $w_{\mathbf{x} \cdot \mathbf{z}}$. Then $w_{\mathrm{x} \cdot \mathbf{z}} \in w_{\lambda \mathbf{y} \cdot(\mathbf{x} \cdot \mathbf{y})} \circ w_{\mathbf{z}}$, but since $\mathbf{x} \cdot \mathbf{z} \oiint_{\Gamma} \lambda \mathrm{y} \cdot(\mathbf{x} \cdot \mathbf{y}) \cdot \mathbf{z}$, $w_{\mathrm{x} \cdot \mathbf{z}} \notin w_{\lambda \mathrm{y} \cdot(\mathbf{x} \cdot \mathrm{y})} \bullet w_{\mathrm{z}}$.

Theorem 3.7. Let $F_{\lambda}$ be the canonical intensional $\lambda$-frame (Definition 3.4) and let $v(\mathrm{r})=\|\mathrm{r}\|$ for any variable or constant $\mathrm{r}$. Then for any term $\mathrm{t}, \llbracket \mathrm{t} \rrbracket^{v}=\|\mathrm{t}\|$.

Proof. By induction on $\mathrm{t}$ we show that $w \in\|\mathrm{t}\|$ (i.e. $\mathrm{t} \in w$ ) if and only if $w \in \llbracket \mathrm{t} \rrbracket^{v}$. Details are in the technical appendix A.3

Lemma 3.8. If there is a valuation $v$ on a frame $F$ such that $\left\{\llbracket \mathrm{t} \rrbracket^{v} \mid \mathrm{t}\right.$ is a term $\}=H$, then $F$ is faithful. Hence the canonical frame $F_{\lambda}$ is faithful.

Proof. See the technical appendix A.4.

Theorem 3.9. $\mathrm{t} \Longrightarrow_{\Gamma} \mathrm{s}$ if and only if $\llbracket \mathrm{t} \rrbracket^{v} \subseteq \llbracket \mathrm{s} \rrbracket^{v}$ for all $\Gamma$-sensitive models.

Proof. The left-right direction is Theorem 2.21

If $\mathrm{t} \bigwedge_{\Gamma} \mathbf{s}$ then, by Theorem 2.7, $\mathrm{t} \varlimsup_{\Gamma} \mathbf{s}$ and so $\mathbf{s} \notin w_{\mathrm{t}}$ in $F_{\lambda}$. Therefore $\|\mathrm{t}\| \nsubseteq\|\mathbf{s}\|$ and so by Theorem 3.7 there is a valuation $v$ such that $\llbracket \mathrm{t} \rrbracket^{v} \nsubseteq \llbracket \mathbb{s} \rrbracket^{v}$ on the canonical frame $F_{\lambda}$. Furthermore, by Lemma 3.8, $F_{\lambda}$ is faithful, and since $\left\|r_{1}\right\| \subseteq\left\|r_{2}\right\|$ for $\left\langle r_{1}, r_{2}\right\rangle \in \Gamma$ it follows that $M_{\lambda}=\left\langle F_{\lambda}, v\right\rangle$ is $\Gamma$-sensitive.

Definition 3.10. An extensional frame is an intensional frame where $\bullet=\circ$, we may define them simply as a triple $\langle W, \bullet, H\rangle$

Corollary 3.11. Let $\Gamma=\left\{\langle\mathrm{t}[\mathrm{x}:-\mathrm{r}], \mathrm{t}[\mathrm{x}:-\lambda \mathrm{y} \cdot(\mathrm{r} \cdot \mathrm{y})]\rangle \mid \mathrm{r} \in \mathcal{L}_{\lambda}\right.$ and $\mathrm{y}$ is not free in $\left.\mathrm{r}\right\}$. Then $\mathrm{t} \Longrightarrow \Longrightarrow_{\Gamma} \mathrm{s}$ if and only if $\llbracket \mathrm{t} \rrbracket^{v} \subseteq \llbracket \mathrm{s} \rrbracket^{v}$ for any faithful extensional frame and any $v$.

Proof. For the left-right direction it is a simple matter to apply the reasoning of Theorem 2.21

For the right-left direction it is enough to note that:

$$
\mathrm{t}[\mathrm{x}:-\mathrm{s} * \mathrm{r}] \underset{(a s s)}{\Longrightarrow_{\Gamma}} \mathrm{t}[:-\lambda \mathrm{y}(\mathrm{s} \cdot \mathrm{y}) * \mathrm{r}] \underset{\left(\beta^{*}\right)}{\Longrightarrow_{\Gamma}} \mathrm{t}[\mathrm{x}:-\mathrm{s} \cdot \mathrm{r}]
$$

so in the construction of the canonical frame $F_{\lambda}$ of Theorem $3.7 . \bullet_{\lambda}=\circ_{\lambda}$. 
Remark 3.12. An extensional frame satisfies $\eta$-expansion. An intensional frame is like an extensional frame except with an additional 'inner' application function. We interpret $\lambda$ in terms of the outer function and application in terms of the inner function to block $\eta$-expansion. $\eta$-expansion will prove very useful in constructing models of $\lambda$-equality in Section 4 But others have also noted good properties and justification in models for $\eta$-expansion JG95.

\section{Equality}

Definition 4.1. Let $\beta=\left\{\langle\mathrm{t}[\mathrm{x}:-\mathrm{s}[\mathrm{y} / \mathrm{r}]], \mathrm{t}[\mathrm{x}:-\lambda \mathrm{y} \cdot \mathrm{s} \cdot \mathrm{r}]\rangle \mid \mathrm{t}, \mathrm{s}, \mathrm{r} \in \mathcal{L}_{\lambda}\right\}$.

Corollary 4.2. When restricted to $\mathcal{L}_{\lambda}, \Longrightarrow_{\beta}$ is the familiar relation of (intensional) $\lambda$-equality, and by Theorem 3.9 is complete for $\beta$-sensitive models.

Corollary 4.2 is itself not so significant as it only tells us half the story about what these models look like, and does not tell us if there are any non-trivial ones. Of course, given independent nontriviality proofs for $\lambda$-equality, ${ }^{1}$ we can use Theorem 3.9 to conclude that there are nontrivial $\beta$-sensitive models. This section is concerned with producing a purely semantic characterisation of $\beta$-sensitivity.

The strategy we shall employ is as follows. First we extend $\mathcal{L}_{\lambda}$ and $\mathcal{L}_{\lambda}^{*}$ with two constant symbols $\mathbf{S}, \mathbf{K}$ and $\mathbf{C}$. We will use these symbols to stand in for constructions involving $\lambda$ expressions. So for example $\mathbf{K} \cdot \mathbf{z}$ will stand in for $(\lambda \mathbf{x} \lambda \mathrm{y} \cdot \mathbf{x}) \cdot \mathbf{z}$, and the former expression contains no instances of $\lambda$. This will allow us to work with certain complex $\lambda$-expressions as if they are free of the symbol $\lambda$ and is particularly useful for a stronger form of induction which we shall use. Then, for each $t$ we describe a new term $[x] t$, built only out of application and the three new constants (effectively the familiar combinator abstraction of [HS08, p.26], but extended to a language that includes the $\lambda$-operator). Then, with the help of the completeness theorem 3.9 , we describe conditions in which a model (or frame) entails that $\llbracket \mathrm{t}[\mathrm{x} / \mathrm{s}] \rrbracket^{v}=\llbracket[\mathrm{x}] \mathrm{t} \cdot \mathrm{s} \rrbracket \rrbracket^{v}$. It then turns out that $\llbracket[\mathrm{x}] \mathrm{t} \rrbracket^{v} \subseteq \llbracket \lambda \mathrm{x} . \mathrm{t} \rrbracket^{v}$ and we thereby obtain models of $\beta$-expansion

Definition 4.3. Let $\mathcal{L}_{\lambda_{C}}^{*}\left(\mathcal{L}_{\lambda_{C}}\right)$ be $\mathcal{L}_{\lambda}^{*}\left(\mathcal{L}_{\lambda}\right)$ with new constant symbols $\mathbf{S}, \mathbf{K}, \mathbf{C}$.

Definition 4.4. When defining or proving a property of a term $t$, we write 'by induction on $(l, \boldsymbol{d})^{\prime}$ to describe an induction on the pair $(l, d)$, lexicographically ordered, where $d$ is the number of occurrences of $\cdot$ in $t$ and $l$ is the number of occurrences of $\lambda$ in $t$.

Definition 4.5. For any $\mathrm{t} \in \mathcal{L}_{\lambda_{C}}$, define $[\mathrm{x}] \mathrm{t}$ by induction on $(l, d)$.

1. (a) $[\mathrm{x}] \mathrm{x}$ is $(\mathbf{S} \cdot \mathbf{K}) \cdot \mathbf{K}$

(b) $[\mathrm{x}] \mathrm{r}$ is $\mathbf{K} \cdot \mathrm{r}$ if $\mathrm{r}$ is a variable or constant distinct from $\mathrm{x}$.

2. $[\mathrm{x}](\mathrm{s} \cdot \mathrm{r})$ is $(\mathbf{S} \cdot[\mathrm{x}] \mathbf{s}) \cdot[\mathrm{x}] \mathrm{r}$

3. $[\mathrm{x}] \lambda z . \mathbf{s}$ is $\mathbf{C} \cdot[\mathrm{z}][\mathrm{x}] \mathbf{s}$

In this definition we allow the case that $\mathrm{z}=\mathrm{x}$ in the clause for $[\mathrm{x}] \lambda \mathrm{z} . \mathrm{s}$.

Lemma 4.6. If $\mathrm{t} \in \mathcal{L}_{\lambda_{C}}$ then $[\mathrm{x}] \mathrm{t} \in \mathcal{L}_{\lambda_{C}}$ and (1) is well defined, (2) contains no instances of $\lambda$, and (3) contains no free occurrences of $\mathrm{x}$.

\footnotetext{
${ }^{1}$ Nontriviality follows syntactically from the Church-Rosser property [HS08 Ch. A2], the cut-elimination theorem of Gab11; and it follows semantically from Scott's famous model $D_{\omega}$ HS08. Ch. 16], among others.
} 
Proof. By induction on $(l, d)$.

Lemma 4.7. Let variable $\mathrm{v}$ not occur in $\mathrm{t}$, then $[\mathrm{x}](\mathrm{t}[\mathrm{y} / \mathrm{v}])=([\mathrm{x}] \mathrm{t})[\mathrm{y} / \mathrm{v}]$

Proof. By induction on $(l, d)$. See the technical appendix A.5

Definition 4.8. Extend the notion of a valuation from Definition 2.11 so that

$$
\llbracket \mathbf{S} \rrbracket^{v}=\llbracket \lambda \mathrm{xyz} \cdot((\mathrm{x} \cdot \mathrm{z}) \cdot(\mathrm{y} \cdot \mathrm{z})) \rrbracket^{v} \quad \llbracket \mathbf{K} \rrbracket^{v}=\llbracket \lambda \mathrm{xy} \cdot \mathrm{x} \rrbracket^{v} \quad \llbracket \mathbf{C} \rrbracket^{v}=\llbracket \lambda \mathrm{xyz} \cdot((\mathrm{x} \cdot \mathrm{z}) \cdot \mathrm{y}) \rrbracket^{v}
$$

Theorem 4.9. If $\mathrm{t} \in \mathcal{L}_{\lambda_{C}}$, then for any $M=\langle F, v\rangle$, $\llbracket[\mathrm{x}] \mathrm{t} * \mathrm{~s} \rrbracket^{v} \subseteq \llbracket \mathrm{t}[\mathrm{x} / \mathrm{s}] \rrbracket^{v}$

Proof. By induction on $(l, d)$. See the technical appendix A.6

Definition 4.10. A model is combinator complete when for any $h_{1}, h_{2}, h_{3} \in H$

$$
\begin{gathered}
\left(h_{1} \bullet h_{3}\right) \bullet\left(h_{2} \bullet h_{3}\right)=\left(\left(\llbracket \mathbf{S} \rrbracket^{v} \bullet h_{1}\right) \bullet h_{2}\right) \bullet h_{3} \quad h_{1}=\left(\llbracket \mathbf{K} \rrbracket^{v} \bullet h_{1}\right) \bullet h_{2} \\
\bigcap_{h \in H}\left\{w \mid w \circ h \subseteq\left(h_{1} \bullet h\right) \bullet h_{2}\right\}=\left(\llbracket \mathbf{C} \rrbracket^{v} \bullet h_{1}\right) \bullet h_{2}
\end{gathered}
$$

A frame $F$ is combinator complete when it is faithful and each $\langle F, v\rangle$ is combinator complete.

Remark 4.11. Notice that no $h \in H$ can be empty if $F$ is a non-trivial combinator complete frame. For if $\varnothing \in H$ then for any $h \in H, h=\left(\llbracket \mathbf{K} \rrbracket^{v} \bullet h\right) \bullet \varnothing=\varnothing$, so then $H=\{\varnothing\}$ and $\llbracket \mathrm{t} \rrbracket^{v}=\llbracket \mathbf{s} \rrbracket^{v}=\varnothing$ for any $\mathrm{t}, \mathrm{s}$ and $v$.

We could have equivalently defined combinator complete frame by requiring that, for any $v$ and any $\mathrm{x}, \mathrm{y}, \mathrm{z}, \llbracket \mathrm{x} \rrbracket^{v}=\llbracket(\mathbf{K} \cdot \mathrm{x}) \cdot \mathrm{y} \rrbracket^{v}, \llbracket(\mathrm{x} \cdot \mathbf{z}) \cdot(\mathrm{y} \cdot \mathrm{z}) \rrbracket^{v}=\llbracket((\mathbf{S} \cdot \mathrm{x}) \cdot \mathrm{y}) \cdot \mathrm{z} \rrbracket^{v}$ and $\llbracket \lambda \mathrm{z} \cdot((\mathrm{x} \cdot \mathrm{z}) \cdot \mathrm{y}) \rrbracket^{v}=$ $\llbracket(\mathbf{C} \cdot \mathbf{x}) \cdot \mathrm{y} \rrbracket^{v}$. But 4.10 is preferable as it is independent of the syntax.

Theorem 4.12. For any combinator complete $\langle F, v\rangle, \llbracket \mathrm{t}[\mathrm{x} / \mathrm{s}] \rrbracket^{v} \subseteq \llbracket[\mathrm{x}] \mathrm{t} \cdot \mathrm{s} \rrbracket^{v}$.

Proof. Again, we proceed by induction on $(l, d)$. See the technical appendix A.7

Corollary 4.13. For any combinator complete model $M, \llbracket \mathrm{t}\left[\mathrm{x} / \mathrm{s} \rrbracket^{v}=\llbracket \lambda \cdot \mathrm{xt} \cdot \mathrm{s} \rrbracket^{v}\right.$.

Proof. Since $\bullet \subseteq \circ$ we have that $\llbracket \lambda x$.t.s $\rrbracket^{v} \subseteq \llbracket \lambda x . t * \mathrm{~s} \rrbracket^{v}$, and so Theorem 4.9 entails that $\llbracket \lambda \mathrm{x} . \mathrm{t} \cdot \mathrm{s} \rrbracket^{v} \subseteq \llbracket \mathrm{t}[\mathrm{x} / \mathrm{s}] \rrbracket^{v}$.

And conversely:

$$
\begin{array}{rlll}
\llbracket \mathrm{t}[\mathrm{x} / \mathrm{s}] \rrbracket^{v} & \subseteq \llbracket[\mathrm{x}] \mathrm{t} \cdot \mathrm{s} \rrbracket^{v} & \text { Thrm } 4.12 \\
& \subseteq \llbracket \lambda \mathrm{x} .([\mathrm{x}] \mathrm{t} * \mathrm{x}) \cdot \mathrm{s} \rrbracket^{v} & \text { Thrm } 3.9, \text { Lemma 2.20 Lemma } 4.6 \\
& \subseteq \llbracket \lambda \mathrm{x} . \mathrm{t} \cdot \mathrm{s} \rrbracket^{v} & \text { Thrm } 4.9
\end{array}
$$

We now have a means of characterising $\beta$-equality semantically.

Corollary 4.14. If $\mathrm{t}, \mathrm{s} \in \mathcal{L}_{\lambda}$ then, $\mathrm{t} \Longrightarrow_{\beta} \mathrm{s}$ iff $\llbracket \mathrm{t} \rrbracket^{v} \subseteq \llbracket \mathrm{s} \rrbracket^{v}$ for all combinator complete models.

Proof. By 4.13 if $\langle\mathrm{t}, \mathrm{s}\rangle \in \beta$ then $\llbracket \mathrm{t} \rrbracket^{v} \subseteq \llbracket \mathrm{s} \rrbracket^{v}$ in all combinator complete models. Furthermore if $\mathrm{t} \Longrightarrow_{\beta} \mathrm{s}$ then $\llbracket \mathrm{t} \rrbracket^{v} \nsubseteq \llbracket \mathrm{s} \rrbracket^{v}$ in the canonical model for $\beta$. It is not hard to verify that the canonical model (moreover, the canonical frame) is combinator complete.

Corollary 4.15. A frame is combinator complete iff it is $\beta$-sensitive 
Definition 4.16. A model is fully extensional when $h=\bigcap_{h^{\prime} \in H}\left\{w \mid w \circ h^{\prime} \subseteq h \bullet h^{\prime}\right\}$ for all $h \in H$.

Remark 4.17. Looking at Definition $2.12 h=\llbracket \mathrm{t} \rrbracket^{v}$ implies $\bigcap_{h^{\prime} \in H}\left\{w \mid w \circ h^{\prime} \subseteq h \bullet h^{\prime}\right\}=$ $\llbracket \lambda \mathrm{y} .(\mathrm{t} \cdot \mathrm{y}) \rrbracket^{v}$ for $\mathrm{y} \notin \mathrm{t}$. So if a model is fully extensional then, for any $\mathrm{t}$ and any $v, \llbracket \mathrm{t} \rrbracket^{v}=$ $\llbracket \lambda \mathrm{x}(\mathrm{t} \cdot \mathrm{x}) \rrbracket^{v}$ for $\mathrm{x}$ not free in $\mathrm{t}$. This implies, by reasoning similar to Corollary 3.11 , that $\bullet=0$.

Definition 4.18. Let $\boldsymbol{\eta}_{e}=\left\{\langle\mathrm{t}[\mathrm{y}:-\mathrm{s}], \mathrm{t}[\mathrm{y}:-\lambda \mathrm{x} .(\mathrm{s} \cdot \mathrm{x})]\rangle \mid \mathrm{r}, \mathrm{s} \in \mathcal{L}_{\lambda}\right.$ and $x$ not free in $\left.\mathbf{s}\right\}$; and let $\boldsymbol{\eta}_{c}=\left\{\langle\mathrm{t}[\mathrm{y}:-\lambda \mathrm{x} .(\mathrm{s} \cdot \mathrm{x})], \mathrm{t}[\mathrm{y}:-\mathbf{s}]\rangle \mid \mathrm{r}, \mathbf{s} \in \mathcal{L}_{\lambda}\right.$ and $x$ not free in $\left.\mathbf{s}\right\} ;$ and finally let $\boldsymbol{\beta} \boldsymbol{\eta}=\boldsymbol{\beta} \cup \boldsymbol{\eta}_{e} \cup \boldsymbol{\eta}_{c}$

Theorem 4.19. If $\mathrm{t}, \mathrm{s} \in \mathcal{L}_{\lambda}$ then, $\mathrm{t} \Longrightarrow \beta \eta \mathrm{s}$ iff $\llbracket \mathrm{t} \rrbracket^{v} \subseteq \llbracket \mathrm{s} \rrbracket^{v}$ for all fully extensional combinator complete models.

Proof. It is straightforward to verify (see Remark 4.17) that $\mathrm{t} \Longrightarrow_{\beta \eta} \mathrm{s}$ implies that $\llbracket \mathrm{t} \rrbracket^{v} \subseteq \llbracket \mathrm{s} \rrbracket^{v}$ in all fully extensional, combinator complete models. Conversely, if $\mathrm{t} \Longrightarrow_{\beta \eta} \mathrm{s}$ then $\llbracket \mathrm{t} \rrbracket^{v} \nsubseteq \llbracket \mathrm{s} \rrbracket^{v}$ in the canonical model for $\beta \boldsymbol{\eta}$, it is not hard to verify that it is combinator complete and fully extensional.

\section{Further Work}

There are numerous other semantics for untyped $\lambda$-calculus, and it is a matter of great interest to compare them to the one given here. A detailed comparison is not possible in this paper, but a few key differences can be noted. The semantics of this paper is complete. Unlike e.g. Scott models [HS08, Ch.16], we interpret the $\lambda$-operator directly. That is, we do not merely present an extensional combinatory algebra that only interprets application and the combinators $\mathbf{S}$ and $\mathbf{K}$. The models and frames of this semantics are ordered, but do not necessarily have a bottom element (the canonical model provides a denotation for each term, and there is no 'bottom' term). Finally, we can distinguish intensional from extensional $\lambda$-equality semantically.

The characterisation of equality offered by Section 4 is still lacking in providing guidance for explicitly constructing a nontrivial model of lambda equality. We know that $\lambda$-calculus is not trivial, so we can use Theorem 3.9 to conclude that such a model exists. But it would be valuable to construct and compare models of intensional and extensional $\lambda$-equality.

Finally, the semantics of this paper potentially provides a new framework for obtaining semantics for 'non-standard' $\lambda$-calculi. By altering the structure of frames and the semantic definition 2.12 we can validate and invalidate different reduction and equality rules. Of particular interest are frames where $H$ is as close to $\mathcal{P}(W)$ as possible, and the behaviour of $\lambda$-terms interpreted in these frames is determined by structural properties of $\bullet$ and $\circ$. This relates to the issue mentioned above of explicitly constructing a nontrivial model within this framework. It is preferable to construct one where $H$ is a simple as possible, perhaps isomorphic to $\mathcal{P}(W)$, (see 4.11 for a reason not to make $H=\mathcal{P}(W)$ ). Carrying such a construction through is a natural next step.

\section{A Technical Appendix}

\section{A.1 Theorem 2.7}

Suppose that $t_{1} \Longrightarrow_{\Gamma} t_{2}$, where $t_{1}, t_{2} \in \mathcal{L}_{\lambda}$. We argue that any such derivation can be converted into a derivation that $t_{1} \longrightarrow_{\Gamma} t_{2}$.

We first argue that any application of $\left(\eta^{*}\right)$ or $(s u b)$ can be pushed in front of (i.e. towards the end of the derivation) an application of any other rule or eliminated entirely. 
- If $\mathbf{s}^{\prime}$ is derived from $\mathbf{s}$ by an application of any rule, then

$$
\mathrm{t}[\mathrm{x}:-\mathrm{s}] \longrightarrow_{\Gamma} \mathrm{t}[\mathrm{x}:-\lambda \mathrm{y} \cdot(\mathrm{s} * \mathrm{y})] \longrightarrow_{\Gamma} \mathrm{t}\left[\mathrm{x}:-\lambda \mathrm{y} \cdot\left(\mathrm{s}^{\prime} * \mathrm{y}\right)\right]
$$

may be replaced by:

$$
\mathrm{t}[\mathrm{x}:-\mathrm{s}] \longrightarrow_{\Gamma} \mathrm{t}\left[\mathrm{x}:-\mathrm{s}^{\prime}\right] \longrightarrow_{\Gamma} \mathrm{t}\left[\mathrm{x}:-\lambda \mathrm{y} \cdot\left(\mathrm{s}^{\prime} * \mathrm{y}\right)\right]
$$

- The case where $t^{\prime}[\mathrm{x}:-\mathrm{s}]$ is derived from $\mathrm{t}[\mathrm{x}:-\mathrm{s}]$ is similar. Except the following three special cases:

1.

$$
\mathrm{t}[\mathrm{x}:-\mathrm{s} \cdot \mathrm{r}] \underset{\left(\eta^{*}\right)}{\longrightarrow} \Gamma[\mathrm{x}:-\lambda \mathrm{y} \cdot(\mathrm{s} * \mathrm{y}) \cdot \mathrm{r}] \underset{(\beta)}{\longrightarrow} \Gamma \mathrm{t}[\mathrm{x}:-\mathrm{s} * \mathrm{r}]
$$

may be replaced by

$$
\mathrm{t}[\mathrm{x}:-\mathrm{s} \cdot \mathrm{r}] \underset{(\mathrm{sub})}{\longrightarrow} \mathrm{t}[\mathrm{x}:-\mathrm{s} * \mathrm{r}]
$$

2.

$$
\mathrm{t}[\mathrm{x}:-\mathrm{s} * \mathrm{r}] \underset{\left(\eta^{*}\right)}{\longrightarrow} \mathrm{t}[\mathrm{x}:-\lambda \mathrm{y} \cdot(\mathrm{s} * \mathrm{y}) * \mathrm{r}] \underset{\left(\beta^{*}\right)}{\longrightarrow} \Gamma[\mathrm{x}:-\mathrm{s} * \mathrm{r}]
$$

may be replaced by

$$
\mathrm{t}[\mathrm{x}:-\mathrm{s} * \mathrm{r}]
$$

3.

$$
\mathrm{t}[\mathrm{x}:-\mathrm{s}] \underset{\left(\eta^{*}\right)}{\longrightarrow_{\Gamma}} \mathrm{t}[\mathrm{x}:-\lambda \mathrm{y} \cdot(\mathrm{s} * \mathrm{y})] \underset{(\alpha)}{\longrightarrow_{\Gamma}} \mathrm{t}[\mathrm{x}:-\lambda \mathrm{z} \cdot(\mathrm{s} * \mathrm{z})]
$$

may be replaced by:

$$
\mathrm{t}[\mathrm{x}:-\mathbf{s}] \underset{\left(\eta^{*}\right)}{\longrightarrow} \mathrm{t}[\mathrm{x}:-\lambda \mathbf{z} \cdot(\mathbf{s} * \mathbf{z})]
$$

- By a similar reasoning it follows that (sub) can be pushed in front of any other rule, with the exception of the special case of the derivation segment

$$
\mathrm{t}[\mathrm{x}:-\lambda \mathrm{y} \cdot \mathrm{s} \cdot \mathrm{r}] \underset{(\text { sub })}{\longrightarrow} \mathrm{t}[\mathrm{x}:-\lambda \mathrm{y} \cdot \mathrm{s} * \mathrm{r}] \underset{\left(\beta^{*}\right)}{\longrightarrow} \Gamma \mathrm{t}[\mathrm{x}:-\mathrm{s}[\mathrm{y} / \mathrm{r}]]
$$

which may be replaced by:

$$
\mathrm{t}[\mathrm{x}:-\lambda \mathrm{y} \cdot \mathrm{s} \cdot \mathrm{r}] \underset{(\beta)}{\longrightarrow_{\Gamma}} \mathrm{t}[\mathrm{x}:-\mathrm{s}[\mathrm{y} / \mathrm{r}]]
$$

It follows that any derivation may be replaced by a derivation where the last application is an instance of $(\eta)$ or $(s u b)$, if either appears in the derivation at all. Since $(\eta)$ and $(s u b)$ introduce an instance of $*$, if $\mathrm{t}_{2} \in \mathcal{L}_{\lambda}$ then no instances of $(\eta)$ or ( $\left.s u b\right)$ occur in the derivation. Furthermore, since $t_{1} \in \mathcal{L}_{\lambda}$ it follows that the derivation contains no instances of $\left(\beta^{*}\right)$ or occurrences of $*$. This implies that $t_{1} \longrightarrow_{\Gamma} t_{2}$. 


\section{A.2 Proof of Theorem 2.16}

We show only $(\beta)$ and $\left(\eta^{*}\right)$ here, the others are equally straightforward.

$$
\begin{aligned}
& \llbracket \lambda \mathrm{x} . \mathrm{t} \cdot \mathrm{s} \rrbracket^{v} \\
& =\llbracket \lambda \mathrm{x} . \mathrm{t} \rrbracket^{v} \bullet \llbracket \mathrm{s} \rrbracket^{v} \quad \text { Definition } 2.12 \\
& =\bigcap_{h \in H}\left\{w \mid w \circ h \subseteq \llbracket \mathbf{t} \rrbracket^{v[\mathbf{x} \mapsto h]}\right\} \bullet \llbracket \mathbf{s} \rrbracket^{v} \quad \text { Definition 2.12 } \\
& \subseteq\left\{w \mid w \circ \llbracket \mathrm{s} \rrbracket^{v} \subseteq \llbracket \mathrm{t} \rrbracket^{v\left[\mathrm{x} \mapsto \llbracket \mathbb{s} \rrbracket^{v}\right]}\right\} \bullet \llbracket \mathrm{t} \rrbracket^{v} \quad \llbracket \mathrm{s} \rrbracket^{v} \in H \\
& \subseteq\left\{w \mid w \bullet \llbracket \mathrm{s} \rrbracket^{v} \subseteq \llbracket \mathrm{t} \rrbracket^{v\left[\mathrm{x} \mapsto \llbracket \mathrm{s} \rrbracket^{v}\right]}\right\} \bullet \llbracket \mathrm{t} \rrbracket^{v} \quad \bullet \subseteq \circ \\
& \subseteq \llbracket \mathrm{t} \rrbracket^{v\left[\mathrm{x} \mapsto \llbracket \mathbb{s}^{v} \rrbracket^{v}\right.} \quad \text { Definition } 2.10 \\
& =\llbracket \phi[\mathrm{x} / \mathrm{s}] \rrbracket^{v} \quad \text { Lemma } 2.15 \\
& \llbracket \mathrm{t} \rrbracket^{v} \subseteq \bigcap_{h \in H}\left\{w \mid w \circ h \subseteq \llbracket \mathrm{t} \rrbracket^{v} \circ h\right\} \quad \text { Definition } 2.10 \\
& =\bigcap_{h \in H}\left\{w \mid w \circ h \subseteq \llbracket \mathrm{t} * \mathrm{x} \rrbracket^{v[\mathrm{x} \mapsto h]}\right\} \quad \mathrm{x} \text { not free in } \llbracket \mathrm{t} \rrbracket^{v} \\
& =\llbracket \lambda \mathrm{x} \cdot(\mathrm{t} * \mathrm{x}) \rrbracket^{v} \\
& \text { Definition } 2.12
\end{aligned}
$$

\section{A.3 Proof of Theorem 3.7}

First, note that $\mathrm{s} \Longrightarrow{ }_{\Gamma}^{\zeta_{f}} \mathrm{r}$ implies $\mathrm{t}[\mathrm{x}:-\mathrm{s}] \Longrightarrow_{\Gamma}^{\zeta_{f}} \mathrm{t}[\mathrm{x}:-\mathrm{r}]$.

Now, we argue by induction on $\mathrm{t}$ we show that $w \in\|\mathrm{t}\|$ (i.e. $\mathrm{t} \in w$ ) if and only if $w \in \llbracket \mathrm{t} \rrbracket^{v}$.

- $\mathrm{t}$ is a variable $\mathrm{x}$. Then $\|\mathrm{x}\|=v(\mathrm{x})=\llbracket \mathrm{x} \rrbracket^{v}$ by the definition of $v$. Similarly, if $\mathrm{t}$ is a constant c.

$-t$ is $t_{1} \cdot t_{2}$.

Suppose $\mathrm{t}_{1} \cdot \mathrm{t}_{2} \in w$, and consider the worlds $w_{\mathrm{t}_{1}}$ and $w_{\mathrm{t}_{2}}$ in $W_{\lambda}$. If $\mathbf{s}_{1} \in w_{\mathrm{t}_{1}}$ and $\mathbf{s}_{2} \in w_{\mathrm{t}_{2}}$ then by Definition 3.3. $\mathrm{t}_{1} \Longrightarrow{ }_{\Gamma}^{\zeta_{f}} \mathrm{~s}_{1}$ and $\mathrm{t}_{2} \Longrightarrow_{\Gamma}^{\zeta_{f}} \mathrm{~s}_{2}$. Thus $\mathrm{t}_{1} \cdot \mathrm{t}_{2} \Longrightarrow{ }_{\Gamma}^{\zeta_{f}} \mathrm{~s}_{1} \cdot \mathrm{s}_{2}$ and $\mathrm{s}_{1} \cdot \mathrm{s}_{2} \in w$. Then by the definition of $\bullet_{\lambda}$ we have that $w \in w_{\mathrm{t}_{1}} \bullet_{\lambda} w_{\mathrm{t}_{2}}$. Furthermore, $w_{\mathrm{t}_{1}} \in\left\|\mathrm{t}_{1}\right\|$ and so by the induction hypothesis, $w_{\mathrm{t}_{1}} \in \llbracket \mathrm{t}_{1} \rrbracket^{v}$. Similarly $w_{\mathrm{t}_{2}} \in \llbracket \mathrm{t}_{2} \rrbracket^{v}$. Hence $w \in \llbracket \mathrm{t}_{1} \cdot \mathrm{t}_{2} \rrbracket^{v}$ by Definition 2.12.

Conversely, suppose that $w \in \llbracket \mathrm{t}_{1} \cdot \mathrm{t}_{2} \rrbracket^{v}$. Then there are $w_{\mathbf{s}_{1}}, w_{\mathbf{s}_{2}}$ such that $w_{\mathbf{s}_{1}} \in \llbracket \mathrm{t}_{1} \rrbracket^{v}$ and $w_{\mathbf{s}_{2}} \in \llbracket \mathrm{t}_{2} \rrbracket^{v}$ and $w \in w_{\mathbf{s}_{1}} \bullet_{\lambda} w_{\mathbf{s}_{2}}$. By the induction hypothesis $w_{\mathbf{s}_{1}} \in\left\|\mathrm{t}_{1}\right\|$ and $w_{\mathbf{s}_{2}} \in\left\|\mathrm{t}_{2}\right\|$. Then $\mathbf{s}_{1} \Longrightarrow{ }_{\Gamma}^{\zeta_{f}} \mathrm{t}_{1}$ and $\mathbf{s}_{2} \Longrightarrow{ }_{\Gamma}^{\zeta_{f}} \mathrm{t}_{2}$. Furthermore, by the construction of $\bullet_{\lambda}, \mathbf{s}_{1} \cdot \mathbf{s}_{2} \in w$ and hence $\mathrm{t}_{1} \cdot \mathrm{t}_{2} \in w$.

$-t$ is $t_{1} * t_{2}$. Similar to the case for .

$-t$ is $\lambda x . s$.

Suppose $\lambda$ x.s $\in w_{1}$. Suppose that $w_{3} \in w_{1} \circ_{\lambda} w_{2}$, and that $w_{2} \in h$ for some $h \in H_{\lambda}$, then $h=\|\mathrm{r}\|$ for some term $\mathrm{r}$. By (ass) and $\zeta_{f}$ we have that $\mathrm{r} \Longrightarrow \Gamma_{\Gamma}^{\zeta_{f}} \mathrm{c}$ and $\mathrm{c} \Longrightarrow_{\Gamma}^{\zeta_{f}} \mathrm{r}$ for some c. So $h=\|\mathbf{c}\|$ and $\mathbf{c} \in w_{2}$. By the construction of $o_{\lambda}, \lambda \mathbf{x} . \mathbf{s} * \mathbf{r} \in w_{3}$ and $\mathbf{s o} \mathbf{s}[\mathrm{x} / \mathrm{c}] \in w_{3}$ by $\left(\beta^{*}\right)$, i.e. $w_{3} \in\|\mathbf{s}[\mathbf{x} / \mathbf{c}]\|$. By the induction hypothesis $\|\mathbf{s}[\mathbf{x} / \mathbf{c}]\|=\llbracket \mathbf{s}[\mathbf{x} / \mathrm{c}] \rrbracket^{v}$. Furthermore by Lemma 2.15 $\llbracket \mathrm{s}[\mathrm{x} / \mathrm{c}] \rrbracket^{v}=\llbracket \mathrm{s} \rrbracket^{v\left[\mathrm{x} \mapsto \llbracket \mathrm{c} \rrbracket^{v}\right]}$. But by the definition of $v, \llbracket \mathrm{c} \rrbracket^{v}=\|\mathrm{c}\|$, and so $w_{3} \in \llbracket \mathbf{s} \rrbracket^{v[\mathrm{x} \mapsto\|\mathrm{c}\|]}$. But $h=\|\mathbf{c}\|$ so $w_{3} \in \llbracket \mathbf{s} \rrbracket^{v[\mathbf{x} \mapsto h]}$. Thus $w_{1} \in\left\{w \mid \forall h \in H_{\lambda} \cdot w \circ_{\lambda} h \subseteq \llbracket \mathbf{s} \rrbracket^{v[\mathbf{x} \mapsto h]}\right\}=\llbracket(\lambda \mathbf{x} . \mathbf{s}) \rrbracket^{v}$. Hence, $\|\lambda \mathrm{x} . \mathrm{s}\| \subseteq \llbracket(\lambda \mathrm{x} . \mathrm{s}) \rrbracket^{v}$

Conversely, suppose that $\lambda \mathrm{x} . \mathrm{s} \notin w_{\mathrm{r}}$ for some $\mathrm{r}$. Let $\mathrm{y}$ be a variable not free in $\mathrm{r}$ or $\mathbf{s}$ and consider the worlds $w_{\mathrm{y}}$ and $w_{\mathrm{r} * \mathrm{y}}$. If $\mathbf{s}[\mathrm{x} / \mathrm{y}] \in w_{\mathrm{r} * \mathrm{y}}$ then $\mathrm{r} * \mathrm{y} \Longrightarrow{ }_{\Gamma}^{\zeta_{f}} \mathbf{s}[\mathrm{x} / \mathrm{y}]$, so $\lambda \mathrm{y} .(\mathbf{r} * \mathrm{y}) \Longrightarrow_{\Gamma}^{\zeta_{f}}$ $\lambda \mathrm{y}(\mathrm{s}[\mathrm{x} / \mathrm{y}])$. But by our choice of $\mathrm{y},(\eta)$ entails that $\mathrm{r} \Longrightarrow_{\Gamma}^{\zeta_{f}} \lambda \mathrm{y} .(\mathrm{r} * \mathrm{y})$. So $\mathrm{r} \Longrightarrow_{\Gamma}^{\zeta_{f}} \lambda \mathrm{y} . \mathrm{s}[\mathrm{x} / \mathrm{y}]$, which contradicts our initial supposition that $\lambda \mathrm{x} . \mathrm{s} \notin w_{\mathrm{r}}$, therefore $\mathrm{s}[\mathrm{x} / \mathrm{y}] \notin w_{\mathrm{r} * \mathrm{y}}$. In other words 
$w_{\mathrm{r} * \mathrm{y}} \notin\|\mathbf{s}[\mathrm{x} / \mathrm{y}]\|$. Therefore, by the induction hypothesis, $w_{\mathrm{r} \cdot \mathrm{y}} \notin \llbracket \mathbf{s}[\mathrm{x} / \mathrm{y}] \rrbracket^{v}$. Since $\llbracket \mathrm{y} \rrbracket^{v}=\|\mathrm{y}\|$, it follows by Lemma 2.15 that $w_{\mathrm{r} \cdot \mathrm{y}} \notin \llbracket \mathbf{s} \rrbracket^{v[\mathbf{x} \mapsto\|\mathrm{y}\|]}$. But clearly $w_{\mathrm{r} * \mathrm{y}} \in w_{\mathrm{r}} \circ_{\lambda} w_{\mathrm{y}}$, so it follows that $w_{\mathrm{r}} \notin\left\{w \mid \forall h \in H_{\lambda} \cdot w \circ_{\lambda} h \subseteq \llbracket \mathbf{s} \rrbracket^{v[\mathrm{x} \mapsto h]}\right\}$. By the semantics of $(\lambda \mathrm{y} . \mathbf{s})$, this means that $w_{\mathrm{r}} \notin \llbracket(\lambda \mathrm{y} . \mathbf{s}) \rrbracket^{v}$. Hence, since every $w \in W_{\lambda}$ is $w_{\mathrm{r}}$ for some $\mathrm{r}, \llbracket(\lambda \mathrm{x} . \mathbf{s}) \rrbracket^{v} \subseteq\|\lambda \mathrm{x} . \mathbf{s}\|$.

\section{A.4 Proof of Lemma 3.8}

We must show that for any valuation $v^{\prime}$ and any term $\mathrm{t}$ that $\llbracket \mathrm{t} \rrbracket^{v^{\prime}} \in H$. By the definition of a valuation $\llbracket \mathrm{x} \rrbracket \rrbracket^{v^{\prime}} \in H$ for any variable $\mathrm{x}$. So if $\llbracket \mathrm{t} \rrbracket^{v^{\prime}} \notin H$ then

$$
\llbracket \mathrm{t} \rrbracket^{v\left[\mathrm{x}_{1} \mapsto \llbracket \mathrm{x}_{1} \rrbracket^{v^{\prime}} \ldots \mathrm{x}_{n} \mapsto \llbracket \mathrm{x}_{n} \rrbracket^{v^{\prime}}\right]} \notin H
$$

where $\mathrm{x}_{1} \ldots \mathrm{x}_{n}$ are the free variables of $\mathrm{t}$. Now, by assumption, $v$ is such that every $h \in H$ is $\llbracket \mathbf{s} \rrbracket^{v}$ for some $\mathbf{s}$. It follows then that we can choose $\mathbf{s}_{1} \ldots \mathbf{s}_{n}$ such that $\llbracket \mathbf{s}_{i} \rrbracket^{v}=v\left[\mathrm{x}_{i} \mapsto \llbracket \mathrm{x}_{i} \rrbracket^{v^{\prime}}\right]$, and so:

$$
\llbracket \mathrm{t} \rrbracket^{v\left[\mathrm{x}_{1} \mapsto \llbracket \mathrm{s}_{1} \rrbracket^{v} \ldots \mathrm{x}_{n} \mapsto \llbracket \mathbf{s}_{n} \rrbracket^{v}\right]} \notin H
$$

This entails, by Theorem 2.15 that $\llbracket \mathrm{t}\left[\mathrm{x}_{i} / \mathrm{s}_{i}\right] \rrbracket^{v} \notin H$. But this contradicts the assumption that $\left\{\llbracket \mathrm{t} \rrbracket^{v} \mid \mathrm{t}\right.$ is a term $\}=H$.

\section{A.5 Proof of Lemma 4.7}

By induction on $(l, d)$.

- If $\mathrm{t}$ is $\mathrm{x}$ then $\mathrm{x}[\mathrm{y} / \mathrm{v}]=\mathrm{x}$ and so

$$
\begin{aligned}
{[\mathrm{x}] \mathrm{x} } & =(\mathbf{S} \cdot \mathbf{K} \cdot) \mathbf{K} & & \text { Def. } 4.5 \\
& =([\mathrm{x}] \mathrm{x})[\mathrm{y} / \mathrm{v}] & & \mathrm{y} \notin(\mathbf{S} \cdot \mathbf{K} \cdot) \mathbf{K}
\end{aligned}
$$

- If $\mathrm{t}$ is $\mathrm{y}$ then $\mathrm{y}[\mathrm{y} / \mathrm{v}]=\mathrm{v}$ and $[\mathrm{x}] \mathrm{y}=\mathbf{K} \cdot \mathrm{y}$. So:

$$
\begin{aligned}
{[\mathrm{x}] \mathrm{v} } & =\mathbf{K} \cdot \mathrm{v} & & \text { Def. } 4.5 \\
& =(\mathbf{K} \cdot \mathrm{y})[\mathrm{y} / \mathrm{v}] & & \mathrm{y} \notin \mathbf{K} \\
& =([\mathrm{x}] \mathrm{y})[\mathrm{y} / \mathrm{v}] & &
\end{aligned}
$$

- The case where $t$ is some variable other than $\mathrm{x}$ or $\mathrm{y}$ is similar.

- If $t$ is $s \cdot r$ then the result follows easily by the induction hypothesis.

- If $t$ is $\lambda z . s$, including the case where $z$ is $x$, then

$$
\begin{array}{rll}
{[\mathrm{x}](\mathrm{t}[\mathrm{y} / \mathrm{v}])} & =\mathbf{C} \cdot[\mathrm{z}][\mathrm{x}](\mathrm{s}[\mathrm{y} / \mathrm{v}]) & \text { Def. } 4.5 \\
& =\mathbf{C} \cdot([\mathrm{z}][\mathrm{x}] \mathrm{s})[\mathrm{y} / \mathrm{v}] & \text { ind. hyp, Lemma } 4.6 \\
& =(\mathbf{C} \cdot[\mathrm{z}][\mathrm{x}] \mathrm{s})[\mathrm{y} / \mathrm{v}] & \mathrm{y} \notin \mathbf{C} \\
& =([\mathrm{x}] \mathrm{t})[\mathrm{y} / \mathrm{v}] &
\end{array}
$$

\section{A.6 Proof of Theorem 4.9}

By induction on $(l, d)$. We appeal to known facts about $\beta$-reduction and the Completeness Theorem 3.9.

$-\mathrm{t}=\mathrm{x}$. Then $[\mathrm{x}] \mathrm{t} * \mathbf{s}$ is $((\mathbf{S} \cdot \mathbf{K} \cdot \mathbf{K}) \cdot \mathbf{s}$, and it is easy to show that for any $M$

$$
\llbracket\left((\mathbf{S} \cdot \mathbf{K} \cdot \mathbf{K}) \cdot \mathbf{s} \rrbracket^{v} \subseteq \llbracket \mathbf{s} \rrbracket^{v}\right.
$$

(see e.g. [HS08, p.26] and use 2.21). And given the validity of $\left(\beta^{*}\right)$ we can also show that $\llbracket\left((\mathbf{S} \cdot \mathbf{K} \cdot \mathbf{K}) * \mathbf{s} \rrbracket^{v} \subseteq \llbracket \mathbf{s} \rrbracket^{v}\right.$ in any $M$. 
- The argument is similar for the case where $\mathrm{t}=\mathrm{y} \neq \mathrm{x}$.

$-\mathrm{t}=\mathrm{t}_{1} \cdot \mathrm{t}_{2}$. Then:

$$
\begin{array}{rlll}
\llbracket\left(\left(\mathbf{S} \cdot[\mathrm{x}] \mathrm{t}_{1}\right) \cdot[\mathrm{x}] \mathrm{t}_{2}\right) * \mathrm{~s} \rrbracket^{v} & \subseteq \llbracket\left([\mathrm{x}] \mathrm{t}_{1} \cdot \mathrm{s}\right) \cdot\left([\mathrm{x}] \mathrm{t}_{2} \cdot \mathrm{s}\right) \rrbracket^{v} & & \text { Def. } 4.8 \\
& \subseteq \llbracket \mathrm{t}_{1}[\mathrm{x} / \mathrm{s}] \cdot \mathrm{t}_{2}[\mathrm{x} / \mathrm{s}] \rrbracket^{v} & \text { Ind. Hyp. } \\
& =\llbracket\left(\mathrm{t}_{1} \cdot \mathrm{t}_{2}\right)[\mathrm{x} / \mathrm{s}] \rrbracket^{v} &
\end{array}
$$

- If $\mathrm{t}$ is $\lambda \mathrm{y} . \mathrm{r}$, then choose a variable $\mathrm{z}$ that does not occur in $\mathrm{r}$ or $\mathbf{s}$. Now, $\mathbf{s}$ and $[\mathrm{x}] \mathrm{s}$ contain fewer instances of $\lambda$ than $t$ (Lemma 4.6), so given Theorem 3.9 we may apply the induction hypothesis as follows:

$$
\begin{aligned}
& \llbracket(\mathbf{C} \cdot[\mathrm{y}][\mathrm{x}] \mathrm{r}) * \mathbf{s} \rrbracket^{v} \subseteq \llbracket \lambda \mathbf{z} \cdot(((\mathbf{C} \cdot[\mathrm{y}][\mathrm{x}] \mathrm{r}) * \mathbf{s}) * \mathbf{z}) \rrbracket^{v} \quad \text { Thrm } 2.21 \\
& \subseteq \llbracket \lambda z \cdot(([\mathrm{y}][\mathrm{x}] \mathrm{r} \cdot \mathrm{z}) * \mathrm{~s}) \rrbracket^{v} \quad \text { Def. } 4.8 \text {, Thrm 3.9 } \\
& \subseteq \llbracket \lambda z .([\mathrm{x}] \mathrm{r}[\mathrm{y} / \mathrm{z}] * \mathrm{~s}) \rrbracket^{v} \quad \text { Ind. Hyp. } \\
& \subseteq \llbracket \lambda \mathrm{z} .(\mathrm{t}[\mathrm{y} / \mathrm{z}, \mathrm{x} / \mathrm{s}]) \rrbracket^{v} \quad \text { Ind. Hyp. } \\
& =\llbracket \lambda \mathrm{y} \cdot(\mathrm{t}[\mathrm{x} / \mathrm{s}]) \rrbracket^{v}
\end{aligned}
$$

\section{A.7 Proof of Theorem 4.12}

We can now proceed by induction on $(l, d)$.

$-\mathrm{t}=\mathrm{x}$. Then $\mathrm{x}[\mathrm{x} / \mathrm{s}]=\mathrm{s}$ and it is not hard to see that the definition of combinator completeness 4.10 implies that $\llbracket \mathbf{s} \rrbracket^{v}=\llbracket((\mathbf{S} \cdot \mathbf{K}) \cdot \mathbf{K}) \cdot \mathbf{s} \rrbracket^{v}$.

- The argument is similar for the case where $t=y \neq x$.

$-\mathrm{t}=\mathrm{t}_{1} \cdot \mathrm{t}_{2}$. Then:

$$
\begin{aligned}
\llbracket\left(\mathrm{t}_{1} \cdot \mathrm{t}_{2}\right)[\mathrm{x} / \mathrm{s}] \rrbracket^{v} & =\llbracket \mathrm{t}_{1}[\mathrm{x} / \mathrm{s}] \rrbracket^{v} \bullet \llbracket \mathrm{t}_{2}[\mathrm{x} / \mathrm{s}] \rrbracket^{v} & & \\
& \subseteq \llbracket\left([\mathrm{x}] \mathrm{t}_{1} \cdot \mathrm{s}\right) \rrbracket^{v} \bullet \llbracket\left([\mathrm{x}] \mathrm{t}_{2} \cdot \mathrm{s}\right) \rrbracket^{v} & & \text { Ind. Hyp. } \\
& =\left(\llbracket[\mathrm{x}] \mathrm{t}_{1} \rrbracket^{v} \bullet \llbracket \mathrm{s} \rrbracket^{v}\right) \bullet\left(\llbracket[\mathrm{x}] \mathrm{t}_{2} \rrbracket^{v} \bullet \llbracket \mathrm{s} \rrbracket^{v}\right) & & \\
& =\left(\left(\llbracket \mathbf{S} \rrbracket^{v} \bullet \llbracket[\mathrm{x}] \mathrm{t}_{1} \rrbracket^{v}\right) \bullet \llbracket[\mathrm{x}] \mathrm{t}_{2} \rrbracket^{v}\right) \bullet \llbracket \mathrm{s} \rrbracket^{v} & & \text { Def } 4.10 \\
& =\llbracket\left(\left(\mathbf{S} \cdot[\mathrm{x}] \mathrm{t}_{1}\right) \cdot[\mathrm{x}] \mathrm{t}_{2}\right) \cdot \llbracket \mathrm{s} \rrbracket^{v} \rrbracket^{v} & & \text { Def. } 2.12 \\
& =\llbracket\left([\mathrm{x}] \mathrm{t}_{1} \cdot \mathrm{t}_{2}\right) \cdot \mathrm{s} \rrbracket^{v} & & \text { Def. } 4.5
\end{aligned}
$$

and the result follows as above.

- Suppose $t$ is $\lambda y . r$. Let $\mathbf{z}$ be chosen so that it does not occur in $t$ or $\mathbf{s}$. Then using Lemma 2.20

$$
\begin{aligned}
\llbracket \lambda \mathrm{y} \cdot(\mathrm{r}[\mathrm{x} / \mathrm{s}]) \rrbracket^{v} & =\llbracket \lambda \mathrm{z} \cdot(\mathrm{r}[\mathrm{y} / \mathrm{z}, \mathrm{x} / \mathrm{s}]) \rrbracket^{v} & & \\
& \subseteq \llbracket \lambda \mathrm{z} \cdot([\mathrm{x}](\mathrm{r}[\mathrm{y} / \mathrm{z}]) \cdot \mathrm{s}) \rrbracket^{v} & & \text { Ind. Hyp. } \\
& =\llbracket \lambda \mathrm{z} \cdot(([\mathrm{x}] \mathrm{r})[\mathrm{y} / \mathrm{z}] \cdot \mathrm{s}) \rrbracket^{v} & & \text { Lemma 4.7 } \\
& \subseteq \llbracket \lambda \cdot((\mathrm{y}][\mathrm{x}] \mathrm{r} \cdot \mathrm{z}) \cdot \mathrm{s}) \rrbracket^{v} & & \text { Ind. Hyp. } \\
& \subseteq \llbracket(\mathbf{C} \cdot[\mathrm{y}][\mathrm{x}] \mathrm{r}) \cdot \mathrm{s} \rrbracket^{v} & & \text { Def. 4.10 } \\
& =\llbracket([\mathrm{x}] \lambda \mathrm{y} \cdot \mathrm{r}) \cdot \mathrm{s} \rrbracket^{v} & & \text { Def. 4.5 }
\end{aligned}
$$

\section{References}

[BBK04] Christoph Benzmüller, Chad E. Brown, and Michael Kohlhase. Higher-order semantics and extensionality. Journal of Symbolic Logic, 69:1027-1088, 2004.

[Gab11] Michael Gabbay. A proof-theoretic treatment of $\lambda$-reduction with cut-elimination: $\lambda$-calculus as a logic programming language. Journal of Symbolic Logic, 76(2):673-699, 2011. 
[GG10] Michael Gabbay and Murdoch James Gabbay. A simple class of kripke-style models in which logic and computation have equal standing. In Edmund M. Clarke and Andrei Voronkov, editors, LPAR (Dakar), volume 6355 of Lecture Notes in Computer Science, pages 231-254. Springer, 2010.

[Hen50] Leon Henkin. Completeness in the theory of types. Journal of Symbolic Logic, 15:81-91, 1950.

[HS08] J. Roger Hindley and Jonathan P. Seldin. Lambda-Calculus and Combinators, An Introduction. Cambridge University Press, 2nd edition, 2008.

[JG95] C. Barry Jay and Neil Ghani. The virtues of eta-expansion. Journal of Functional Programming, 5(2):135-154, April 1995.

[Sel03] Peter Selinger. Order-incompleteness and finite lambda reduction models. Theoretical Computer Science, 309(1):43-63, 2003.

[Sha00] Stewart Shapiro. Foundations without foundationalism: a case for second-order logic. Number 17 in Oxford logic guides. Oxford University Press, 2000. 